\title{
Semilattices global valuations in the topos approach to quantum mechanics
}

\author{
H. Freytes ${ }^{1,2}$, C. De Ronde ${ }^{3,4,5}$, G. Domenech ${ }^{4}$
}

1. Dipartimento di Filosofia, Università di Cagliari

Viale Merello 92, 09123, Cagliari - Italia.

2. Departamento de Matemática UNR-CONICET

Av. Pellegrini 250, CP 2000 Rosario - Argentina.

3. Instituto de Filosofía "Dr. Alejandro Korn" UBA-CONICET

Puan 480, Buenos Aires - Argentina.

4. Center Leo Apostel (CLEA)

5. Foundations of the Exact Sciences (FUND)

Brussels Free University Krijgskundestraat 33, 1160 Brussels - Belgium

\begin{abstract}
In the framework of the topos approach to quantum mechanics a kind of global valuation is introduced and studied. It allows us to represent certain features related to the logical consequences of properties about quantum systems when its phase space is endowed with an intuitionistic strucure.

Keywords: Intuitionistic quantum logic, modal operators, global valuations. Mathematics Subject Classification: 81P10, 81P13, 03G25.
\end{abstract}

\section{Introduction}

Quantum Mechanics (QM) seems to be producing, since approximately the last two decades, a new technological and experimental quantum era. Quantum computation, quantum cryptography, quantum teleportation and the sort are starting a radical change in the way in which we process information. But while quantum information processing is giving rise the most outstanding developments we still lack a coherent physical representation of the theory that would allow us to explain what is QM really talking about. 
One of the main separation lines that draws a clear distinction between the many interpretations of QM is the "positive" or "negative" attitude towards the main features of the formalism. In between them quantum contextuality rises as one the main characteristics that needs to be carefully considered.

It is well known that in classical physics every system can be described by specifying its actual properties. Mathematically, this happens by representing the state of the system by a point $(p, q)$ in the corresponding phase space $\Gamma$ and its properties by subsets of $\Gamma$, with a structure of operations compatible with the usual mathematics of set theory. Consequently, the propositional structure associated with the properties of a classical system follows the rules of classical logic. In the orthodox formulation of QM, a pure state of a system is represented by a ray in the Hilbert space $\mathcal{H}$ and its physical properties by closed subspaces of $\mathcal{H}$, which with adequate definitions of meet and join operations give rise to an orthomodular lattice [24]. This lattice, denoted by $\mathcal{L}(\mathcal{H})$, is called the Hilbert lattice associated to $\mathcal{H}$ and motivates the standard quantum logic or orthomodular quantum logic introduced in the thirties by Birkhoff and von Neumann [1]. But while in classical physics any situation allows an interpretation in terms of sets of actual (preexistent) properties, QM forbids such an interpretation due to the contextual character of the theory. Let us analyze this in detail.

Assigning values to a physical quantity $\mathcal{M}$ is equivalent to establishing a Boolean homomorphism $v: W_{\mathbf{M}} \rightarrow \mathbf{2}$. Thus, we can say that it makes sense to use the "classical discourse" - this is, the classical logical laws are valid - within the context given by $\mathcal{M}$. One may define a global valuation of the physical magnitudes over $\mathcal{L}(\mathcal{H})$ as a family of Boolean homomorphisms $\left(v_{i}: W_{i} \rightarrow \mathbf{2}\right)_{i \in I}$ such that $v_{i}\left|W_{i} \cap W_{j}=v_{j}\right| W_{i} \cap W_{j}$ for each $i, j \in I$, being $\left(W_{i}\right)_{i \in I}$ the family of Boolean sublattices of $\mathcal{L}(\mathcal{H})$. This global valuation would give the values of all magnitudes at the same time maintaining a compatibility condition in the sense that whenever two magnitudes shear one or more projectors, the values assigned to those projectors are the same from every context. Kochen-Specker (KS) Theorem rules out this possibility [21]. In algebraic terms, this theorem is expressed as follows $[4,5]$

Theorem If $\mathcal{H}$ is a Hilbert space such that $\operatorname{dim}(\mathcal{H})>2$, then a global valuation over $\mathcal{L}(\mathcal{H})$ is not possible.

This impossibility to assign values to the properties simultaneously satisfying compatibility conditions is a weighty obstacle for the interpretation of the formalism. The problem of contextuality has been studied from dif- 
ferent approaches. One of them is the modal algebraic approach version related to partial valuations of the orthomodular lattice of closed subspaces of Hilbert space developed in $[6,7,11]$. This proposal allows to identify the constraints imposed by the structure to the relation between actuality and possibility and the discourse that includes both type of propositions. Orthomodular lattices enriched with a modal operator constitute the algebraic framework for this approach. A different approach to contextuality is the one proposed by Isham and Döring which takes quantum systems as modeled by a topos [8, 9]. In this framework, projectors are mapped to sub-objects of the spectral presheaf, a process they call daseinisation which means "bringing-a-quantum-proposition-into-existence" [8]. The set of sub-objects of the spectral sheaf forms a Heyting algebra. From a logical point of view, a nice feature of this approach is that - as any topos - the topos in which the quantum theory is represented comes with an intrinsic intuitionistic logic.

In this work we introduce and study a kind of global valuation for the quantum systems whose logical structure is encoded in a Hilbert lattice $\mathcal{L}(\mathcal{H})$. These global valuations only preserve the bounded join-semilattice structure of $\mathcal{L}(\mathcal{H})$. These valuations are motivated by a modal extension of the intuitionistic structure of quantum properties when the topos approach to $\mathrm{QM}$ is considered. In this modal extension, modal operators encode the notion of logical consequence of a physical property associated to a quantum system modeled by a topos [12]. Let us note that this new type of modal approach has a different interpretation with respect to the algebraic modal extensions in orthomodular structures aforementioned above. Then, semilattice global valuations will allow us to describe features related to the logical consequences of physical properties.

The paper is organized as follows: In Section 1, we recall some basic notions about orthomodular lattices, Boolean algebras and Heyting algebras. In Section 2, we describe the topos approach to quantum systems and the intuitionistic structrure of quantum phase space. In Section 3 a modal extension and a type of classical interpretation for quantum systems are introduced. This modal extension is conceived from the intuitionistic structure of the phase space. In Section 4 a set of global valuations based on the bounded semilattice structure preservation of Hilbert lattices are defined. These valuations represent algebraic properties related to the modal extension of the intuitionistic structure of the phase space. Finally, in Section 5 these bounded semilatice valuations are interpreted. 


\section{Basic Notions}

Now we recall from $[2,20,24]$ some notions of universal algebra and lattice theory that will play an important role in what follows. Let $\mathcal{L}$ be a bounded lattice with 0 the minimum element and 1 the maximum element. If $a \in \mathcal{L}$, an element $c \in \mathcal{L}$ is said to be a complement of $a$ iff $a \wedge c=0$ and $a \vee c=1$. Let $\mathcal{L}=\langle\mathcal{L}, \vee, \wedge, 0,1\rangle$ be a bounded lattice. Given $a, b, c$ in $\mathcal{L}$, we write: $(a, b, c) D$ iff $(a \vee b) \wedge c=(a \wedge c) \vee(b \wedge c) ;(a, b, c) D^{*}$ iff $(a \wedge b) \vee c=(a \vee c) \wedge(b \vee c)$ and $(a, b, c) T$ iff $(a, b, c) D,(\mathrm{a}, \mathrm{b}, \mathrm{c}) D^{*}$ hold for all permutations of $a, b, c$. An element $z$ of a lattice $\mathcal{L}$ is called central iff for all elements $a, b \in \mathcal{L}$ we have $(a, b, z) T$ and $z$ is complemented. We denote by $Z(\mathcal{L})$ the set of all central elements of $\mathcal{L}$ and it is called the center of $\mathcal{L}$. If $\mathcal{L}$ is a bounded lattice then $Z(\mathcal{L})$ is a Boolean sublattice of $\mathcal{L}[24$, Theorem 4.15].

A lattice with involution $[19]$ is an algebra $\langle\mathcal{L}, \vee, \wedge, \neg\rangle$ such that $\langle\mathcal{L}, \vee, \wedge\rangle$ is a lattice and $\neg$ is a unary operation on $\mathcal{L}$ that fulfills the following conditions: $\neg \neg x=x$ and $\neg(x \vee y)=\neg x \wedge \neg y$. An orthomodular lattice is an algebra $\langle\mathcal{L}, \wedge, \vee, \neg, 0,1\rangle$ of type $\langle 2,2,1,0,0\rangle$ that satisfies the following conditions

1. $\langle\mathcal{L}, \wedge, \vee, \neg, 0,1\rangle$ is a bounded lattice with involution,

2. $x \wedge \neg x=0$.

3. $x \vee(\neg x \wedge(x \vee y))=x \vee y$

We denote by $\mathcal{O} \mathcal{M L}$ the variety of orthomodular lattices. It is well known that if $\mathcal{H}$ is a Hilbert space then $\mathcal{L}(\mathcal{H})$, the lattice of closed subspaces of $\mathcal{H}$, also called Hilbert lattice, is an orthomodular lattice. Boolean algebras are orthomodular lattices satisfying the distributive law $x \wedge(y \vee z)=(x \wedge$ $y) \vee(x \wedge z)$. We denote by 2 the Boolean algebra of two elements.

Let $B$ be a Boolean algebra. A subset $I \subseteq B$ is an ideal iff it satisfies: if $a \in I$ and $x \leq a$ then $x \in I$ and if $a, b \in I$ then $a \vee b \in I . I$ is a proper ideal iff $I \neq B$ or, equivalently, $1 \notin I$. If $X \subseteq B$, the ideal $I_{X}$ generated by $X$ is the minimum ideal containing $X$. It is well know that $I_{X}=\left\{x \in B: \exists x_{1} \cdots x_{n} \in X\right.$ with $\left.x_{1} \vee \cdots \vee x_{n} \geq x\right\}$. Each ideal $I$ in $B$ determines univocally a congruence in which the equivalence classes are given by $[x]=\{y \in B: \neg x \vee y \in I$ and $x \vee \neg y \in I\}$. In this case the quotient set $B / \sim$, noted as $B / I$, is a Boolean algebra and the natural application $x \mapsto[x]$ is a Boolean homomorphism form $B$ onto $B / I$. A proper ideal $I$ is maximal iff the quotient algebra $B / I$ is isomorphic to 2 . If 
$f: B \rightarrow \mathbf{2}$ is a Boolean homomorphisms then the preimage of 0 , i.e. $f^{-1}(0)$ is a maximal ideal. Dualizing the definition of ideal yields the definition of filter. In this way, all results obtained for ideals can be obtained for filters applying duality.

A Heyting algebra [2] is an algebra $\langle A, \vee, \wedge, \rightarrow, 0\rangle$ of type $\langle 2,2,2,0\rangle$ satisfying the following equations:

$\mathrm{H} 1\langle A, \vee, \wedge, 0\rangle$ is a lattice with universal lower bound 0 ,

$\mathrm{H} 2 x \wedge y=x \wedge(x \rightarrow y)$,

H3 $x \wedge(y \rightarrow z)=x \wedge((x \wedge y) \rightarrow(x \wedge z))$,

$\mathrm{H} 4 z \wedge((x \wedge y) \rightarrow x)=z$.

We denote by $\mathbb{H}$ the variety of Heyting algebras. In agreement with the usual Heyting algebraic operations, we define the negation $\neg_{H} x=x \rightarrow 0$ and $1=\neg_{H} 0$.

In each Heyting algebra $A$, the reduct $\langle A, \vee, \wedge, 0,1\rangle$ is a bounded distributive lattice. The lattice order, expressed in terms of the operation $\rightarrow$, is equivalent to $a \leq b$ iff $1=a \rightarrow b$. Moreover, for $a, b \in A, a \rightarrow b=$ $\bigvee\{x \in A: x \wedge a \leq b\}$. Boolean algebras are Heyting algebras satisfying the equation $x \vee \neg_{H} x=1$. In this case, the operation $\rightarrow$ satisfies that $x \rightarrow y=\neg_{H} x \vee y$. In a Heyting algebra $A$ the set of central elements, which is a Boolean subalgebra of $A$, is given by $Z(A)=\left\{z \in A: z \vee \neg_{H} z=1\right\}$.

\section{The phase space for quantum systems}

The main idea of this approach is to represent propositions about the values of physical quantities with sub-objects of the phase space of the system, just as in classical physics propositions are represented by subsets of the state space $\Gamma$. This does not mean that the mentioned 'values' are classical values, may be they are not even real numbers, but it is required that the associated sub-objects form some sort of logic, just as the subsets of $\Gamma$ form a Boolean algebra.

In the present paper, we continue previous research [12] in which propositions were represented by modal operators in a Heyting algebra. The encoding of physical properties in a Heyting algebra provides an intuitionistic description of phase space. In fact, when a quantum system is represented 
by a von Neumann algebra $N$, the abelian subalgebras of $N$ represent contexts in which, restricted to the context, the rules of classical logic hold (see for discussion [4]). This algebraic formulation of QM usually starts with the $C^{*}$-algebra of observables. This is a complex algebra $A$ that is complete in a norm $\|\cdot\|$ satisfying $\|x y\| \leq\|x\|\|y\|$ and has an unary involutive operation * such that $\left\|x^{*} x\right\|=\|x\|^{2}$. In this way, a quantum system is mathematically modeled by a $C^{*}$-algebra. If $\mathcal{H}$ is a Hilbert space, the algebra $B(\mathcal{H})$ of all bounded operators of $\mathcal{H}$, equipped with the usual norm and adjoint is an example of $C^{*}$-algebra. By the Gelfand-Naimark theorem [?], any $C^{*}$ algebra is isomorphic to a norm-closed self-adjoint subalgebra of $B(\mathcal{H})$ for some Hilbert space $\mathcal{H}$.

A von Neumann algebra $N$ is a special case of $C^{*}$-algebra $N \subseteq B(\mathcal{H})$ equal to its own bicommutant. More precisely, if $N^{\prime}$ is the set of all bounded operators on $\mathcal{H}$ that commute with every element of $N$ then $N^{\prime \prime}=N$. Whereas $C^{*}$-algebra are usually considered in their norm-topology, a von Neumann algebra carries in addition a second interesting topology, called the weak-topology, in which it is complete as well. In this topology, one has convergence $x_{n} \rightarrow x$ iff, for each density operator $\rho, \operatorname{tr} \rho\left(x_{n}-x\right) \rightarrow 0$ in $\mathcal{H}$ where $t r$ is the trace. A general $C^{*}$-algebra may not have any nontrivial projections while a von Neumann algebra is generated by its projections, i.e., elements satisfying $p^{2}=p^{*}=p$. In a von Neumann algebra, the projections are in natural correspondence with the closed subspaces of a Hilbert space. In this way, projections of a von Neumann algebra form a complete orthomodular lattice. A state in a von Neumann algebra $N$ is a linear functional $s: N \rightarrow \mathbb{C}$ that is continuous in the weak topology and such that $s\left(x^{*} x\right) \geq 0$ and $s(1)=1$.

In the usual topos approach $[9,17,14]$ physical properties are encoded in a Heyting algebra. This provides an intuitionistic description for the phase space of the system. More precisely, in a quantum system represented by a von Neumann algebra $N$, the abelian subalgebras of $N$ represent contexts in which, restricted to the context, the rules of classical logic hold (see for discussion [4]). Let $N$ be a von Neumann algebra and $\mathcal{V}(N)$ be a family of commutative subalgebras of $N$ which share the unit element with $N$. Consider the partial ordered set $\langle\mathcal{V}(N), \subseteq\rangle$ viewed as the small category whose arrows are defined by the partial order $\subseteq$. In the topos approach the system is modeled in the category of presheaves

$$
\widehat{\mathcal{V}(N)}=\operatorname{Set}^{\mathcal{V}(N)^{o p}}
$$


Thus, the category $\widehat{\mathcal{V}(N)}$ can be seen as a category of sets fibred over the contexts. Let $N$ be an abelian von Neumann algebra. A multiplicative state is a state $s$ such that $s(x y)=s(x) s(y)$. We denote by $\Sigma(N)$ the set of multiplicative states in $N$ and the weak ${ }^{*}$ topology is considered in $\Sigma(N)$. We recall that if a classical system is modeled as an abelian von Neumann algebra $N, \Sigma(N)$ represents the phase space of the system.

To model a quantum system, the spectral presheaf is defined as the functor:

$$
\Sigma: \mathcal{V}(N)^{o p} \rightarrow \text { Set }
$$

such that, $\mathcal{V}(N) \ni A \mapsto \Sigma(A)$ and, for each arrow $f: A \rightarrow B$, (i.e., $f$ is the inclusion $A \subseteq B), \Sigma(f)$ is the function $\Sigma(f): \Sigma(B) \rightarrow \Sigma(A)$ such that $(\Sigma(f))(s)=\left.s\right|_{A}$ is naturally chosen as the state space.

Let $N$ be an abelian von Neumann algebra and $\mathcal{P}(N)$ be the set of projections. Let $P \in \mathcal{P}(N)$. It is well known that the set $C_{P}=\{s \in$ $\Sigma(N): s(P)=1\}$ is clopen when the weak* topology is considered in $\Sigma(N)$. Moreover, if we consider the set $C l p(\Sigma(N))$ of all clopen sets in $\Sigma(N)$, the function $C: \mathcal{P}(N) \rightarrow C l p(\Sigma(N))$ such that $C(P)=C_{P}$ is a bijection. A clopen subobject of the spectral presheaf $\Sigma$ is a subfunctor $T$ of $\Sigma$ such that for each $A \in \mathcal{V}(N), T(A) \in C l p(\Sigma(N))$.

When considering $S u b_{c l}(\Sigma)$, the set of clopen subobjects of $\Sigma$, we can see that, $S u b_{c l}(\Sigma)$ is a bounded distributive lattice where the operations $\vee, \wedge$ over clopen subobjects are defined pointwise in each subalgebra of $\mathcal{V}(N), 0$ is the empty subobject and $1=\Sigma$. In $[8, \S 2.3]$ and $[9$, Theorem 2.5] the following result is proved:

Theorem 2.1 $S u b_{c l}(\Sigma)$ is a complete Heyting algebra.

In a classical system, represented by a commutative von Neumann algebra, the subsets of the phase space with usual set operations define the logical (Boolean) structure of the system. For a quantum system, represented by a von Neumann algebra whose phase space is modeled by the spectral presheaf $\Sigma, S u b_{c l}(\Sigma)$ represents the logical structure of the system which is intuitionistic. We will reefer to $S u b_{c l}(\Sigma)$ as the the algebra of propositions associated to the spectral presheaf $\Sigma$.

\section{A modal extension for $S u b_{c l}(\Sigma)$}

In the framework of orthomodular logic, a classical proposition is usually represented by a Boolean (also called central) element of an orthomodular 
lattice $[5, ?]$. In particular, propositions about classical systems are represented by a Boolean algebra. Suppose that $\mathcal{L}$ is a lattice representing the propositional structure associated to a quantum system. A classical interpretation of $\mathcal{L}$ implies assuming that each $x \in \mathcal{L}$ has a classical complement $\neg_{c} x$ and satisfies distributivity conditions in this interpretation. Then, if $x$ is not a classical proposition in $\mathcal{L}$, a classical interpretation of $\mathcal{L}$ must, al least, endow a complement for $x$. Thus, a natural way to algebraically represent classical interpretations are embeddings of $\mathcal{L}$ into Boolean algebras, preserving lattice order structure. In [12] the following general formalization of the concept of classical interpretation for quantum properties encoded in $S u b_{c l}(\Sigma)$ is proposed.

Definition 3.1 Let $S u b_{c l}(\Sigma)$ be the algebra of propositions associated to the spectral presheaf $\Sigma$. A classical interpretation of the properties about the system is a $\vee, \wedge, 0,1$ - embedding $\mathcal{C}: S u b_{c l}(\Sigma) \hookrightarrow B$ where $B$ is a Boolean algebra.

To study this type of classical interpretation, we use the theory of modal operators on Heyting algebras which has its main application in the theory of topoi and sheafication [10,22].

Let $A$ be a Heyting algebra and $a, b \in A$. We say that $b$ is a logical consequence of $a$ iff $a \leq b$ or equivalently $1=a \rightarrow b$. We denote by $[a$ ) the set of logical consequences of $a$. We remark that $[a)$ is the principal filter associated to $a$ in $A$. A modal operator on $A[23]$ is a unary operation $j$ such that:

$$
x \leq j(x), \quad j j(x)=j(x), \quad j(x \wedge y)=j(x) \wedge j(y) .
$$

Let $A$ be a Heyting algebra and $a \in A$. Two interesting modal operators are

$$
\diamond_{a}(x)=a \vee x, \quad \diamond_{a \rightarrow}(x)=a \rightarrow x .
$$

$\diamond_{a}(x)$ is known as closed modal operator and $\diamond_{a \rightarrow}(x)$ is known as open modal operator. In [12, Proposition 5.3] it is proved that

Proposition 3.2 Let $A$ be a Heyting algebra and $a, b \in A$. Then

1. $\operatorname{Imag}\left(\diamond_{a}\right)=[a)$,

2. $\diamond_{\neg_{H} a}(x) \leq \diamond_{a \rightarrow}(x)$,

3. $\operatorname{Imag}\left(\diamond_{a \rightarrow}\right) \subseteq \operatorname{Imag}\left(\diamond_{\neg_{H} a}\right)=\left[\neg_{H} a\right)$, 
4. $a$ is a Boolean element in $A$ iff $\diamond_{\neg_{H} a}=\diamond_{a \rightarrow \text {. }}$.

The set $M(A)$ of all modal operators on $A$ is partially ordered by the relation

$$
j_{1} \leq j_{2} \text { iff } j_{1}(x) \leq j_{2}(x) \text {, for all } x \in A .
$$

If $A$ is a complete Heyting algebra, this partial order defines a complete Heyting algebra structure on $M(A)$ [23, Theorem 2.3] where $\bigwedge_{i} j_{i}$ is given by the operation $\left(\bigwedge_{i} j_{i}\right)(x)=\bigwedge_{i} j_{i}(x)$. The implication $j_{1} \rightarrow j_{2}$ is given by the operation $\left(j_{1} \rightarrow j_{2}\right)(x)=\bigwedge\left\{j_{1}(y) \rightarrow j_{2}(y): y \geq x\right\}$. Joins in $M(A)$ are defined as $j_{1} \vee j_{2}=\bigwedge\left\{j \in M(A): j_{1}, j_{2} \leq j\right\}$.

Theorem $3.3[18, \S 2.6, \S 2.7]$ Let $A$ be a complete Heyting algebra and $a \in A$ then:

1. $\diamond_{a}$ is a Boolean element in $M(A)$ and $\diamond_{a \rightarrow}$ is its complement in $M(A)$.

2. The map $a \mapsto \diamond_{a}$ defines an injective $\langle\bigvee, \wedge, 0,1\rangle$-homomorphism $A \rightarrow$ $\operatorname{Reg}(M(A))$.

3. $a \mapsto \diamond_{a}$ is an isomorphism iff $A$ is a Boolean algebra.

In general, $a \mapsto \diamond_{a}$ does not preserve the operation $\rightarrow$ exception made in the case in which $A$ is a Boolean algebra.

Definition 3.4 Let $A$ be a complete Heyting algebra. We define the algebra $A^{\diamond}$ as the Boolean subalgebra of $\operatorname{Reg}(M(A))$ generated by the following set $\left\{\diamond_{a}, \diamond_{a \rightarrow}: a \in A\right\}$.

When considering the properties of the system encoded in $S u b_{c l}(\Sigma)$, the lattice embedding $\mathcal{C}_{0}: S u b_{c l}(\Sigma) \rightarrow S u b_{c l}(\Sigma)^{\diamond}$ such that $\mathcal{C}_{0}(a)=\diamond_{a}$ can be seen as a classical interpretation of the quantum properties. Suppose that $a$ is a quantum property encoded in $S u b_{c l}(\Sigma)$. Then, by Proposition 3.2-1, the classical interpretation of $a$, given by the modal operator $\diamond_{a}$, makes reference to the logical consequences of $a$ in $S u b_{c l}(\Sigma)$. The Boolean complement of $a$ in $S u b_{c l}(\Sigma)^{\diamond}$ given by $\diamond_{a \rightarrow}$, by Theorem 3.3-1 and Proposition 3.2-3, makes reference only to the consequences of $\neg_{H} a$ in $S u b_{c l}(\Sigma)$ that have the form 
$a \rightarrow x$. This means that, thinking of $a$ as a classical property forces us to only consider as the consequences of $\neg_{H} a$ those of the form $a \rightarrow x$.

Note that if $a \vee \neg_{H} a=1$ then $\diamond_{\neg_{H}} a=\diamond_{a \rightarrow}$. This means that the encoding of physical properties in $S u b_{c l}(\Sigma)$, by Proposition 3.2-4, a classical property is distinguished from a non classical one via the form of the logical consequences of its intuitionistic negation in $S u b_{c l}(\Sigma)$.

The classical interpretation $\mathcal{C}_{0}: S u b_{c l}(\Sigma) \rightarrow S u b_{c l}(\Sigma)^{\diamond}$ may be associated to a piece of the classical language that describes some facts regarding the intuitionistic logical consequences of the propositions about the system. Thus, the classical interpretation given by $\mathcal{C}_{0}$ describes semantic aspects of the intuitionistic logic of phase spaces.

\section{Daseinisation and semilattices global valuations}

The notion of daseinisation is introduced in [8] in order to establish a relation between a physical property about the system and each possible context. Let $\mathcal{H}$ be a Hilbert space and $\mathcal{V}\left(N_{\mathcal{H}}\right)$ the family of all unital commutative von Neumann sub algebras of $\mathcal{B}(\mathcal{H})$ ordered by inclusion and viewed as a category poset. Let $P \in \mathcal{L}(\mathcal{H})$ i.e. a projector that represent a propositions about the system and $V \in \mathcal{V}\left(N_{\mathcal{H}}\right)$. Then we define

$$
\delta(P)_{V}=\bigwedge_{Q \in \mathcal{P}(V): Q \geq P} Q
$$

Note that $\delta(P)_{V} \in V$ and $\delta(P)_{V}$ is considered as an approximation of $P$ from above in the context $V$. In particular if $P \in V$ then $\delta(P)_{V}=P$. Thus, the set $C_{\delta(P)_{V}}=\left\{s \in \Sigma(V): s\left(\delta(P)_{V}\right)=1\right\}$ is a clopen in the weak* topology of $\Sigma(V)$. In [8, Theorem 2.4] it is proved that, for each $P \in \mathcal{L}(\mathcal{H})$ the set $\delta(P)=\left\{C_{\delta(P)_{V}}: V \in \mathcal{V}\left(N_{\mathcal{H}}\right)\right\}$ defines a clopen subobject of $\Sigma$ establishing an embedding

$$
\delta: \mathcal{L}(H) \rightarrow S u b_{c l}(\Sigma) \text { s.t. } P \mapsto \delta(P)
$$

named daseinisation. The function $\delta$ preserves the operations $\langle\vee, 0,1\rangle$ i.e. it is a bounded $\vee$-semilattice homomorphism.

Let us consider the classical interpretation $\mathcal{C}_{0}: S u b_{c l}(\Sigma) \hookrightarrow S u b_{c l}(\Sigma)^{\diamond}$ in

the sense of Definition 3.1. The composition $\mathcal{L}(\mathcal{H}) \stackrel{\delta}{\rightarrow} S u b_{c l}(\Sigma) \stackrel{\mathcal{C}_{0}}{\rightarrow} S u b_{c l}(\Sigma)^{\diamond}$ also defines a bounded $\vee$-semilattice homomorphism. Since it is always possible to establish a Boolean homomorphism $v: S u b_{c l}(\Sigma)^{\diamond} \rightarrow \mathbf{2}$ we can consider de following commutative diagram: 


$$
\begin{gathered}
\mathcal{L}(\mathcal{H}) \stackrel{\widehat{v}}{\longrightarrow} \mathbf{2} \\
\mathcal{C}_{0} \delta \downarrow \\
S u b_{c l}(\Sigma)^{\diamond}
\end{gathered}
$$

Note that $\widehat{v}$ acts as a kind of global valuation assigning truth and falsity to all propositions about the system. Unlike the notion of global valuation given in Section 2, $\widehat{v}$ is another type of valuation that does not preserve the orthomodular structure $\mathcal{L}(\mathcal{H})$. In fact, $\widehat{v}$ is a bounded $\vee$-semilattice homomorphism. It motivates the following notion of global valuation based on semilattices homomorphisms.

Definition 4.1 Let $\mathcal{L}(H)$ be a Hilbert lattice. A global $\diamond$-valuation is a bounded $\vee$-semilattice homomorphism $\widehat{v}: \mathcal{L}(H) \rightarrow \mathbf{2}$ factorizable as in Diagram (4).

We are interested in characterizing and interpreting the bounded $\vee$ semilattice homomorphisms $\widehat{v}: \mathcal{L}(H) \rightarrow \mathbf{2}$ defining global $\diamond$-valuations. Note that, the bounded $\vee$-semilattice embedding $\mathcal{C}_{0} \delta: \mathcal{L}(\mathcal{H}) \rightarrow S u b_{c l}(\Sigma)^{\diamond}$ can be see as the assignment $P \mapsto \diamond_{P}$ where $\diamond_{P}$ make reference to the logical consequences of $P$ in $S u b_{c l}(\Sigma)$. By the $\vee$-preservation of $\mathcal{C}_{0} \delta$ we identify the join of $\mathcal{L}(H)$ with the join of $S u b_{c l}(\Sigma)^{\diamond}$ and then

$$
\diamond_{P \vee Q}=\diamond_{P} \vee \diamond_{Q}
$$

Since $\delta(P \wedge Q) \leq \delta(P) \wedge \delta(Q)$, the infimum $\delta(P) \wedge \delta(Q)$ is not itself of the form $\delta(R)$ where $R \in \mathcal{L}(H)$. Note that

$$
\diamond_{P \wedge Q} \leq \diamond_{P} \wedge_{\Sigma} \diamond_{Q}
$$

where $\wedge_{\Sigma}$ is the infimum in $S u b_{c l}(\Sigma)^{\diamond}$.

Theorem 4.2 Let $\mathcal{L}(H)$ be a Hilbert lattice and $\widehat{v}: \mathcal{L}(H) \rightarrow \mathbf{2}$ be a bounded $\checkmark$-semilattice homomorphism. Then the following conditions are equivalent:

1. $\widehat{v}$ is a global $\diamond$-valuation.

2. If $\widehat{v}(P)=0$ and $\widehat{v}\left(P_{1}\right)=\ldots=\widehat{v}\left(P_{n}\right)=1$ then $\diamond_{P_{1}} \wedge_{\Sigma} \ldots \wedge_{\Sigma} \diamond_{P_{n}} \not \leq \diamond_{P}$. 
Proof: $\quad 1 \Longrightarrow 2$ ) Suppose that $\widehat{v}$ is a global $\diamond$-valuation and let $v$ : $S u b_{c l}(\Sigma)^{\diamond} \rightarrow \mathbf{2}$ be a Boolean homomorphisms such that $\widehat{v}=v\left(\mathcal{C}_{0} \delta\right)$. By $[2$, Theorem $4, \S$ III.3] $v^{-1}(1)$ is a maximal filter $S u b_{c l}(\Sigma)^{\diamond}$ and by the factorization of $\widehat{v}$, form each $P \in \mathcal{L}(H), \diamond_{P} \in v^{-1}(1)$ iff $\widehat{v}(P)=1$. Thus, if $\widehat{v}(P)=0$ (i.e. $\left.\diamond_{P} \notin v^{-1}(1)\right)$ and $\widehat{v}\left(P_{1}\right)=\ldots=\widehat{v}\left(P_{n}\right)=1$ (i.e. $\diamond_{P_{1}} \ldots \diamond_{P_{n}} \in v^{-1}(1)$ ) then, $\diamond_{P_{1}} \wedge_{\Sigma} \cdots \wedge_{\Sigma} \diamond_{P_{n}} \not \leq \diamond_{P}$.

$2 \Longrightarrow 1)$ Since $\widehat{v}$ is bounded $\vee$-semilattice homomorphism, $\widehat{v}^{-1}(0)$ is closed by $\vee$. Then, for each finite subset $\left\{P_{1} \ldots P_{n}\right\} \subseteq \widehat{v}^{-1}(0), \bigvee_{i=1}^{n} P_{i}<1$. The composition $\mathcal{L}(\mathcal{H}) \stackrel{\mathcal{C}_{0} \delta}{\rightarrow} S u b_{c l}(\Sigma)^{\diamond}$ is an injective bounded $\vee$-semilattice homomorphism therefore $\bigvee_{i=1}^{n} \diamond_{P_{i}}=\bigvee_{i=1}^{n} \mathcal{C}_{0} \delta\left(P_{i}\right)=\mathcal{C}_{0} \delta\left(\bigvee_{i=1}^{n} P_{i}\right)<1$ It proves that the ideal $I_{\widehat{v}^{-1}(0)}^{\diamond}$ in $S u b_{c l}(\Sigma)^{\diamond}$, generated by $\mathcal{C}_{0} \delta\left(\widehat{v}^{-1}(0)\right)$, is not trivial i.e., $I_{\widehat{v}^{-1}(0)}^{\diamond} \neq S u b_{c l}(\Sigma)^{\diamond}$. Let $F_{\widehat{v}^{-1}(1)}^{\diamond}$ be the filter in $S u b_{c l}(\Sigma)^{\diamond}$ generated by $\left\{\diamond_{P}: \widehat{v}(P)=1\right\}$. By hypothesis we can see that $F_{\widehat{v}^{-1}(1)}$ is a not trivial filter.

We shall prove that $F_{\widehat{v}^{-1}(1)}^{\diamond} \cap I_{\widehat{v}^{-1}(0)}^{\diamond}=\emptyset$. Suppose that $x \in F_{\widehat{v}^{-1}(1)}^{\diamond} \cap$ $I_{\widehat{v}^{-1}(0)}$. By definition of generated ideal and generated filter in a Boolean algebra there exists $Q_{1} \ldots Q_{m}$ in $\widehat{v}^{-1}(0)$ and $P_{1} \ldots P_{n}$ in $\widehat{v}^{-1}(1)$ such that

$$
\diamond_{P_{1}} \wedge_{\Sigma} \ldots \wedge_{\Sigma} \diamond_{P_{n}} \leq x \leq \bigvee_{i=1}^{m} \diamond_{Q_{i}}=\bigvee_{i=1}^{m} \mathcal{C}_{0} \delta\left(Q_{i}\right)=\mathcal{C}_{0} \delta\left(\bigvee_{i=1}^{m} Q_{i}\right)
$$

Note that, $\bigvee_{i=1}^{m} Q_{i}=P \in \mathcal{L}(H), \widehat{v}(P)=\widehat{v}\left(\bigvee_{i=1}^{m} Q_{i}\right)=\bigvee_{i=1}^{m} \widehat{v}\left(Q_{i}\right)=0$ and $\diamond_{P_{1}} \wedge_{\Sigma} \ldots \wedge_{\Sigma} \diamond_{P_{n}} \leq \diamond_{P}$ which is a contradiction. Hence $F_{\widehat{v}^{-1}(1)}^{\diamond} \cap I_{\widehat{v}^{-1}(0)}^{\diamond}=\emptyset$. By the Prime Ideal Theorem [2, Theorem 1, $\S$ III.4], there exists a maximal ideal $I_{M}$ in $S u b_{c l}(\Sigma)^{\diamond}$ such that $I_{\widehat{v}^{-1}(0)}^{\diamond} \subseteq I_{M}$ and $I_{M} \cap F_{\widehat{v}^{-1}(1)}^{\diamond}=\emptyset$. Then the natural boolean homomorphism $v: S u b_{c l}(\Sigma)^{\diamond} \rightarrow S u b_{c l}(\Sigma)^{\diamond} / I_{M} \approx \mathbf{2}$ is such that $v\left(\diamond_{P}\right)=0$ iff $\widehat{v}=0$. It proves that $\widehat{v}=v\left(\mathcal{C}_{0} \delta\right)$ and $\widehat{v}$ is a global $\diamond$-valuation.

\section{Interpreting global $\diamond$-valuations}

Let $\mathcal{H}$ be a Hilbert space representing a quantum system and $\mathcal{V}\left(N_{\mathcal{H}}\right)$ be the family of all unital commutative von Neumann sub algebras of $\mathcal{B}(\mathcal{H})$ ordered by inclusion. When we consider the phase space described by the spectral presheaf $\Sigma$ and the propositional structure intuitionistically modeled by the Heyting algebra $S u b_{c l}(\Sigma)$, Theorem 4.2 provides an interpretation for global $\diamond$-valuations. 
By Eq.(1) and Eq.(2) an inequality in $S u b_{c l}(\Sigma)^{\diamond}$ of the form

$$
\diamond_{P_{1}} \wedge_{\Sigma} \ldots \wedge_{\Sigma} \diamond_{P_{n}} \leq \diamond_{P}
$$

expresses that for each $x \in S u b_{c l}(\Sigma)$,

$$
\begin{aligned}
\left(P_{1} \vee x\right) \wedge_{\Sigma} \ldots \wedge_{\Sigma}\left(P_{n} \vee x\right) & =\left(P_{1} \wedge_{\Sigma} \ldots \wedge_{\Sigma} P_{n}\right) \vee x \\
\leq & P \vee x
\end{aligned}
$$

or equivalently $P_{1} \wedge_{\Sigma} \cdots \wedge_{\Sigma} P_{n} \leq P$ in $S u b_{c l}(\Sigma)$.

Thus, by Theorem 4.2, a global $\diamond$-valuation $\widehat{v}$ describes a situation in which, if $\widehat{v}(P)=0$ and $\widehat{v}\left(P_{1}\right)=\ldots=\widehat{v}\left(P_{n}\right)=1$, then $P$ can not be a logical consequence in $S u b_{c l}(\Sigma)$ of $P_{1} \wedge_{\Sigma} \ldots \wedge_{\Sigma} P_{n}$. An equivalent way of saying the same thing is the following:

Let $F_{\widehat{v}^{-1}(1)}$ be the filter in $S u b_{c l}(\Sigma)$ generated by $\{P: \widehat{v}(P)=1\}$ i.e. the intuitionistic theory axiomatized by $\{P: \widehat{v}(P)=1\}$. Then, Theorem 4.2 says that: $\widehat{v}(P)=0$ iff $P \notin F_{\widehat{v}^{-1}(1)}$. In other words, the elements $P \in \mathcal{L}(H)$ such that $\widehat{v}(P)=0$ are not intuitionistic logical consequences of the theory $F_{\widehat{v}^{-1}(1)}$.

By Eq.(6) we also note that, under the hypothesis $\widehat{v}(P)=0$ and $\widehat{v}\left(P_{1}\right)=$ $\ldots=\widehat{v}\left(P_{n}\right)=1$, it could occur that $P_{1} \wedge \ldots \wedge P_{n} \leq P$ in $\mathcal{L}(H)$. In other words, $P$ could be a logical consequence of $P_{1}, \ldots, P_{n}$ in the sense of the standard quantum logic $\mathcal{L}(H)$ but not an intuitionistic logical consequence of $P_{1}, \ldots, P_{n}$ in the sense of $S u b_{c l}(\Sigma)$.

Thus, global $\diamond$-valuations are kinds of global valuations in the standard quantum logic $\mathcal{L}(H)$ providing information concerning the logical con-

sequences of propositions about the system encoded in the intuitionistic structure $S u b_{c l}(\Sigma)$.

\section{Conclusions}

In this paper we have introduced global $\diamond$-valuations and studied the features it implies regarding the logical properties about a quantum system when its phase space is endowed with an intuitionistic structure.

\section{References}

[1] G. Birkhoff and J. von Neumann, "The logic of quantum mechanics", Ann. Math. 37 (1936) 823-843 . 
[2] R. Balbes and Ph. Dwinger, Distributive Lattices, University of Missouri Press, Columbia, 1974.

[3] S. Burris, H. P. Sankappanavar, A Course in Universal Algebra, Graduate Text in Mathematics, Vol. 78. Springer-Verlag, New York Heidelberg Berlin, 1981.

[4] C. de Ronde, H., Freytes, G. Domenech, "Interpreting the Modal Kochen-Specker Theorem: Possibility and Many Worlds in Quantum Mechanics", Stud. Hist. Phil. Mod. Phys. 45 (2014) 11-18.

[5] G. Domenech, H. Freytes, "Contextual logic for quantum systems", J. Math. Phys. 46 (2005) 012102.

[6] G. Domenech, H. Freytes, C. de Ronde "Scopes and limits of modality in quantum mechanics" Annalen der Physik 15 (2006) pp 853-860.

[7] G. Domenech, H. Freytes, C. de Ronde "Many worlds and modality in the interpretation of quantum mechanics: an algebraic approach" J. Math. Phys. 50 (2009) 072108.

[8] A. Döring and C.J. Isham, "A topos foundation for theories of physics: II. Daseinisation and the liberation of quantum theory", J. Math. Phys. 49, (2008) 053516.

[9] A. Döring and C.J. Isham, "What is a thing?: topos theory in the foundations of physics" in New Structures for Physics, Lecture Notes for Physics 813, B. Coecke (Editor), Springer, Berlin-Heidelberg, 2010, 753-937.

[10] P. J. Freyd, "Aspects of Topoi". Bull. Austral. Math. Soc. 7 (1972) $1-76$.

[11] H. Freytes, C. de Ronde, G. Domenech, "Modal type othomodular logic" Mathematical Logic Quarterly 55 No 3 (2009), pp 287-299.

[12] H. Freytes, C. de Ronde, G. Domenech, "Physical Properties as Modal Operators in the Topos Approach to Quantum Mechanics", Foundations of Physics 44, Issue 12, (2014) pp 1357-1368.

[13] C. Heunen, N. Landsman, B. Spitters and S. Wolters, "The Gelfand spectrum of a noncommutative $\mathrm{C}^{*}$-algebra: a topos theoretic approach", J. Australian Math. Soc. 90 (2011) 39-52. 
[14] C. Heunen, N. Landsman and B. Spitters, "A topos for algebraic quantum theory", Comm. Math. Phys. 291 (2009) 63-110.

[15] A. Heyting, "Die formalen Regeln der intuitionistischen Logik". Die Preussische Akademie der Wissenschaften. Sitzungsberichte. Physikalische-Mathematische Klasse, 42-56 (1930).

[16] A. Heyting, "Die formalen Regeln der intuitionistischen Mathematik II, III". Die Preussische Akademie der Wissenschaften. Sitzungsberichte. Physikalische-Mathematische Klasse., 57-71, 158-169 (1930).

[17] C. Isham, "Topos methods in the foundations of physics", in Deep beauty: understanding the quantum world through mathematical innovation, H. Halvorson (Editor), Cambridge University Press, 2010, 187206.

[18] P. T. Johnstone, Stone Spaces, Cambridge Studies in Advanced Mathematics 3, Cambridge University Press, Cambridge, 1982.

[19] Kalman, J. A., "Lattices with involution", Trans. Amer. Math. Soc. 87, 485-491 (1958).

[20] Kalmbach, G., Ortomodular Lattices (Academic Press, London, 1983).

[21] Kochen, S. and Specker, E., "On the problem of hidden variables in quantum mechanics", J. Math. Mech. 17, 59-87 (1967).

[22] F.W. Lawvere, "Quantifiers and Sheaves". Actes Congres Intern. Math. (1970), Tome 1, 329-334.

[23] D.S. Macnab, "Modal operators on Heyting algebras", Alg. Univ. 12 (1981) 5-29

[24] F. Maeda and S. Maeda, Theory of symetric lattices, Springer-Verlag, Berlin, 1970.

[25] E. Zafiris and V. Karakostas, "A categorial semantic representation of quantum event structures", Found. Phys. 43 (2013) 1090-1123. 